\title{
THE NON-NULL DISTRIBUTION OF THE LIKELIHOOD RATIO CRITERION FOR ADDITIONAL INFORMATION HYPOTHESIS IN CANONICAL CORRELATION ANALYSIS
}

\author{
Akio Suzukawa* and Yoshiharu Sato**
}

\begin{abstract}
This paper deals with the likelihood ratio test for a hypothesis concerning the relevance of a subset of variables from each of two given variable sets in a canonical correlation analysis (CCA). This hypothesis can be considered as having two conditions for the covariance structure. Assuming that one of these two conditions holds, we obtain the exact moments of the likelihood ratio criterion and the asymptotic expansions of the power function of the likelihood ratio test.
\end{abstract}

\section{Introduction}

McKay [5] has previously described an additional information hypothesis in CCA when one of the two given variable sets is augmented by adding an additional variable set to it. Under the conditions when each of the two given variable sets is augmented, Fujikoshi [2] outlined an additional information hypothesis in CCA for generalization of the hypothesis discussed in McKay [5]. These hypotheses are useful for variable selection in CCA. In a conditional set-up, the testing problem of the hypothesis formulated by McKay [5] is equivalent to independence testing for two variable sets. Therefore, the likelihood ratio criterion for testing the McKay [5] hypothesis and its null and non-null distributions can be obtained from the test of independence. (see, Anderson [1] Chapter 9, Muirhead [6] Chapter 11).

The likelihood ratio criterion for testing the Fujikoshi [2] hypothesis and the asymptotic expansion of its null distribution are obtained by Fujikoshi [2]. Additionally unbiasedness of the likelihood ratio test has been proved by Kariya, Fujikoshi and Krishnaiah [4]. Because no other the results concerning this testing problem have been reported, we consider here the non-null distribution of the likelihood ratio criterion. Under restricted alternatives, this can be obtained in the same way as the non-null distribution of the likelihood ratio criterion for independence testing of two variable sets. The restriction is placed for the covariance strucure, and the non-null distributions obtained in this paper are distributions under the intermediate hypotheses between the null hypothesis and the alternative hypothesis.

\section{Additional information hypothesis}

Let $\boldsymbol{x}=\left[\boldsymbol{x}_{u}^{\prime}, \boldsymbol{x}_{v}^{\prime}\right]^{\prime}$ be random vector with means $\boldsymbol{\mu}=\left[\boldsymbol{\mu}_{u}^{\prime}, \boldsymbol{\mu}_{v}^{\prime}\right]^{\prime}$ and a nonsin-

Received February, 1995. Revised November, 1995. Accepted January, 1996.

* Department of Information Sciences, Faculty of Science and Technology, Science University of Tokyo ; Yamazaki 2641, Noda, Chiba, 278, Japan.

** Division of Information Engineering, Graduate School of Hokkaido University ; Kita 13, Nishi 8, Kita-ku, Sapporo, 060, Japan. 
gular covariance matrix

$$
\Sigma=\left[\begin{array}{ll}
\Sigma_{u u} & \Sigma_{u v} \\
\Sigma_{v u} & \Sigma_{v v}
\end{array}\right]
$$

where $x_{u}$ and $x_{v}$ have $p$ and $q$ components respectively. We partition $x_{u}$ into two subvectors with $p_{1}$ and $p_{2}$ components, respectively, and $x_{v}$ into two subvectors with $q_{1}$ and $q_{2}$ components, respectively ; that is $\boldsymbol{x}_{u}=\left[\boldsymbol{x}_{1}^{\prime}, \boldsymbol{x}_{2}^{\prime}\right]^{\prime}, \boldsymbol{x}_{v}=$ $\left[\boldsymbol{x}_{3}^{\prime}, \boldsymbol{x}_{4}^{\prime}\right]^{\prime}$. The mean vectors $\boldsymbol{\mu}$ and the covariance matrix $\Sigma$ are partitioned similarly,

$$
\boldsymbol{\mu}=\left[\begin{array}{l}
\mu_{1} \\
\mu_{2} \\
\mu_{3} \\
\mu_{4}
\end{array}\right], \quad \Sigma=\left[\begin{array}{llll}
\Sigma_{11} & \Sigma_{12} & \Sigma_{13} & \Sigma_{14} \\
\Sigma_{21} & \Sigma_{22} & \Sigma_{23} & \Sigma_{24} \\
\Sigma_{31} & \Sigma_{32} & \Sigma_{33} & \Sigma_{34} \\
\Sigma_{41} & \Sigma_{42} & \Sigma_{43} & \Sigma_{44}
\end{array}\right] .
$$

Let $\rho_{j}$ and $\left[\boldsymbol{\alpha}_{u j}^{\prime} \boldsymbol{x}_{u}, \boldsymbol{\alpha}_{v j}^{\prime} \boldsymbol{x}_{v}\right]$ be the $j$ th canonical correlation and the $j$ th pair of the canonical variates between $\boldsymbol{x}_{u}$ and $\boldsymbol{x}_{v}$, respectively. Corresponding to partitions of $x_{u}$ and $x_{v}$, we partition $\boldsymbol{\alpha}_{u j}$ and $\boldsymbol{\alpha}_{v j}$ as $\boldsymbol{\alpha}_{u j}=\left[\boldsymbol{\alpha}_{1 j}^{\prime}, \boldsymbol{\alpha}_{2 j}^{\prime}\right]^{\prime}$ and $\boldsymbol{\alpha}_{v j}=\left[\boldsymbol{\alpha}_{3 j}^{\prime}\right.$, $\left.\boldsymbol{\alpha}_{4 j}^{\prime}\right]^{\prime}$. Let $\rho_{j}^{*}$ be $j$ th canonical correlation between $x_{1}$ and $x_{3}$. From Fujikoshi $[2]$, it holds that

$$
\rho_{j} \geq \rho_{j}^{*}, \quad j=1, \cdots, m^{*} \text { and } m \geq m^{*},
$$

where $m=\operatorname{rank}\left(\Sigma_{u v}\right)$ and $m^{*}=\operatorname{rank}\left(\Sigma_{13}\right)$. This inequality implies that each canonical correlation does not decrease by adding $x_{2}$ to $x_{1}$ and $x_{4}$ to $x_{3}$. Fujikoshi [2] has defined a hypothesis of [ $\left.\boldsymbol{x}_{2}^{\prime}, \boldsymbol{x}_{4}^{\prime}\right]$ in CCA between $\boldsymbol{x}_{u}$ and $\boldsymbol{x}_{v}$ that gives no additional information by

$$
H: \boldsymbol{\alpha}_{2 j}=\boldsymbol{o}, \quad \boldsymbol{\alpha}_{4 j}=\boldsymbol{o} \quad(j=1, \cdots, m) .
$$

Hypothesis $H$ can be interpreted as that where the subset $\left[x_{2}^{\prime}, x_{4}^{\prime}\right]$ supplies no additional information about the linear relationship between $x_{u}$ and $x_{v}$, indepen. dently of $\left[\boldsymbol{x}_{1}^{\prime}, \boldsymbol{x}_{3}^{\prime}\right]$.

Let $\boldsymbol{X}_{1}, \cdots, \boldsymbol{X}_{N}$ be a random sample of the size $N=n+1(>p+q)$ of $\boldsymbol{x}=\left[\boldsymbol{x}_{u}^{\prime}\right.$, $\left.\boldsymbol{x}_{v}^{\prime}\right]^{\prime}$, and

$$
\overline{\boldsymbol{X}}=\frac{1}{N} \sum_{t=1}^{N} \boldsymbol{X}_{t}, \quad \boldsymbol{W}=\sum_{t=1}^{N}\left(\boldsymbol{X}_{t}-\overline{\boldsymbol{X}}\right)\left(\boldsymbol{X}_{t}-\overline{\boldsymbol{X}}\right)^{\prime}
$$

We partition $\boldsymbol{W}$ corresponding the partition of $\boldsymbol{\Sigma}$. Fujikoshi [2] has previously proven the following theorem.

THEOREM 1. (Fujikoshi [2]) The following four statements hold.

( $i)$ Each of the following conditions $(a),(b),(c)$ and $(d)$ is equivalent to the hypothesis $H$ defined by (2.2).

(a) $\rho_{j}=\rho_{j}^{*}, \quad j=1, \cdots, m^{*}$ and $m=m^{*}$

(b) $\Sigma_{2 v \cdot 1}=O$ and $\Sigma_{41 \cdot 3}=0$

(c) $\Sigma_{4 u \cdot 3}=\boldsymbol{O}$ and $\Sigma_{23 \cdot 1}=\boldsymbol{O}$ 


\section{(d) $\quad \Sigma_{24 \cdot 13}=O, \quad \Sigma_{23.1}=O$ and $\quad \Sigma_{41 \cdot 3}=O$}

(ii) Let $\boldsymbol{x}=\left[\boldsymbol{x}_{u}^{\prime}, \boldsymbol{x}_{v}^{\prime}\right]^{\prime}$ be distributed according to $N_{p+q}(\boldsymbol{\mu}, \Sigma)$. Then the likelihood ratio criterion $\lambda$ for $H$ is given by

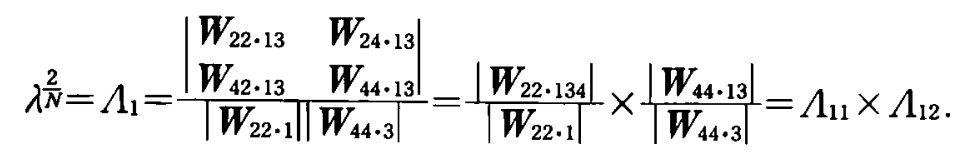

(iii) When $H$ is true, the statistics $\Lambda_{11}$ and $\Lambda_{12}$ in (2.3) are independently distributed according to $\Lambda\left(p_{2}, q, n-p_{1}-q\right)$ and $\Lambda\left(q_{2}, p_{1}, n-p_{1}-q_{1}\right)$, respectively, where $\Lambda(p, q, n)$ denotes the distribution of $\Lambda=|\boldsymbol{A}| /|\boldsymbol{A}+\boldsymbol{B}|$, when $\boldsymbol{A}$ and $\boldsymbol{B}$ are independently distributed according to $W_{p}(n, I)$ and $W_{p}(q, \boldsymbol{I})$, respectively.

(iv) The null distribution of the likelihood ratio criterion for $H$ given by (2.3) can be approximated asymptotically up to the order $M^{-1}$ by

$$
\operatorname{Pr}(-2 \rho \log \lambda \leq x)=\operatorname{Pr}\left(\chi_{f}^{2} \leq x\right)+O\left(M^{-2}\right),
$$

where $f=p q-p_{1} q_{1}$,

$$
\rho=1-\frac{1}{2 N}\left\{p+q+3-\frac{1}{f} p_{1} q_{1}\left(p_{2}+q_{2}\right)\right\},
$$

and $M=\rho N$.

The sign of the last term in the correction factor $m$ in Fujikoshi [2] Theorem 3 should be positive. We corrected this in (2.5).

From condition (a), the equality holds in (2.1) if and only if $H$ is true. Hence, if hypothesis $H$ is true, we can say that the subset $\left[\boldsymbol{x}_{2}^{\prime}, \boldsymbol{x}_{4}^{\prime}\right]$ is redundant in CCA between $\boldsymbol{x}_{u}$ and $\boldsymbol{x}_{v}$. Condition (d) is useful for obtaining the likelihood ratio criterion for $H$. From condition (b), we can see that hypothesis $H$ requires both the redundancy of $x_{2}$ in CCA between $x_{u}$ and $x_{v}$ and the redundancy of $\boldsymbol{x}_{4}$ in CCA between $\boldsymbol{x}_{1}$ and $\boldsymbol{x}_{v}$. Because the condition $\boldsymbol{\Sigma}_{2 v \cdot 1}=\boldsymbol{O}$ is equivalent to the no additional information hypothesis of $\boldsymbol{x}_{2}$ in CCA between $\boldsymbol{x}_{u}$ and $\boldsymbol{x}_{v}$, condition $\boldsymbol{\Sigma}_{41 \cdot 3}=\boldsymbol{O}$ is equivalent to the no additional information hypothesis of $\boldsymbol{x}_{4}$ in CCA between $\boldsymbol{x}_{1}$ and $\boldsymbol{x}_{v}$. Condition (c) can be interpreted similarly.

\section{The non-null distribution of the likelihood ratio criterion}

The non-null distribution of the likelihood ratio criterion $\lambda$ is considered in this section. It is difficult to obtain the distribution of $\lambda$ under the general alternative however, because of the dependency of decomposed $\Lambda$-variates such as $\Lambda_{11}$ and $\Lambda_{12}$ in (2.3). Consequently we will consider this under the intermediate hypothesis between the null and the alternative, which is a hypothesis restricted by the condition that $\boldsymbol{x}_{2}$ is redundant in CCA between $\boldsymbol{x}_{u}$ and $\boldsymbol{x}_{v}$.

The likelihood ratio criterion $\lambda$ given by (2.3) can be interpreted as follows. Our problem is testing for

$$
H: \Sigma_{2 v \cdot 1}=O, \Sigma_{41 \cdot 3}=O \quad \text { v.s. } K: \Sigma_{2 v \cdot 1} \neq O, \Sigma_{41 \cdot 3} \neq O \text {. }
$$


We consider the intermediate hypothesis between $H$ and $K$;

$$
H_{1}: \Sigma_{2 v \cdot 1}=\boldsymbol{O}, \Sigma_{41 \cdot 3} \neq O \text {. }
$$

Then, the likelihood ratio statistics for $H$ v.s. $H_{1}$ and for $H_{1}$ v.s. $K$ are given by $\Lambda_{12}$ and $\Lambda_{11}$, respectively. The following lemma implies that under $H_{1}, \Lambda_{12}$ and $\Lambda_{11}$ are independent, and the distribution of $\Lambda_{11}$ does not depend on unknown parameters. Hence, if the distribution of $\Lambda_{12}$ under $H_{1}$ can be derived, we can obtain the distribution of $\Lambda_{1}=\Lambda_{11} \times \Lambda_{12}$ under $H_{1}$. Based on this idea, we consider the distribution of $\Lambda_{1}$ under the intermediate hypothesis $H_{1}$.

LEMMA 1. Concerning the distributions of $\Lambda_{11}$ and $\Lambda_{12}$ defined in (2.3), the following three statements hold under $H_{1}$.

(i) $\Lambda_{11}$ and $\Lambda_{12}$ are independently distributed.

(ii) $\Lambda_{11}$ is distributed according to $\Lambda\left(p_{2}, q, n-p_{1}-q\right)$.

(iii) We denote the distribution of $\Lambda=|\boldsymbol{A}| /|\boldsymbol{A}+\boldsymbol{B}|$ by $\Lambda(p, q, n, \boldsymbol{\Omega})$, when $\boldsymbol{A}$ and $\boldsymbol{B}$ are independently distributed according to $W_{p}(n, \boldsymbol{I})$ and $W_{p}(q, \boldsymbol{I}$, $\boldsymbol{\Omega})$, respectively. When $q_{2} \leq p_{1}$, the conditional distribution of $\Lambda_{12}$ given $\tilde{\boldsymbol{W}}_{11 \cdot 3}$ which is distributed according to $W_{p_{1}}\left(n-q_{1}, \boldsymbol{I}_{p_{1}}\right)$ is $\Lambda\left(q_{2}, p_{1}, n-p_{1}\right.$ $\left.-q_{1}, \Omega\right)$ where $\lambda_{1}^{2}, \cdots, \lambda_{q_{2}}^{2}$ are the ordered latent roots of $\boldsymbol{\Sigma}_{44 \cdot 3}^{-1} \boldsymbol{\Sigma}_{41 \cdot 3} \Sigma_{11 \cdot 3}^{-1} \Sigma_{14 \cdot 3}$,

$$
\tilde{\boldsymbol{P}}\left(q_{2} \times p_{1}\right)=\left[\begin{array}{cccc}
\lambda_{1} & & \boldsymbol{O} & \\
& \ddots & \vdots & \boldsymbol{O} \\
\boldsymbol{O} & & \lambda_{q_{2}} &
\end{array}\right]=\left[\begin{array}{l}
\boldsymbol{P} \vdots \boldsymbol{O}
\end{array}\right], \quad \boldsymbol{\Phi}=\boldsymbol{I}_{q_{2}}-\boldsymbol{P}^{2}
$$

and $\boldsymbol{\Omega}=\boldsymbol{\Phi}^{-1} \tilde{\boldsymbol{P}} \tilde{\boldsymbol{W}}_{11 \cdot 3} \tilde{\boldsymbol{P}}^{\prime}$.

When $q_{2}>p_{1}$, the conditional distribution of $\Lambda_{12}$ given $\tilde{W}_{44 \cdot 3}$, which is distributed according to $W_{q_{2}}\left(n-q_{1}, \boldsymbol{I}_{q_{2}}\right)$ is $\Lambda\left(p_{1}, q_{2}, n-q, \boldsymbol{\Omega}^{*}\right)$, where $\lambda_{1}^{2}, \cdots$, $\lambda_{p_{1}}^{2}$ are the ordered latent roots of $\Sigma_{11 \cdot 3}^{-1} \Sigma_{14 \cdot 3} \Sigma_{44 \cdot 3}^{-1} \Sigma_{41 \cdot 3}$,

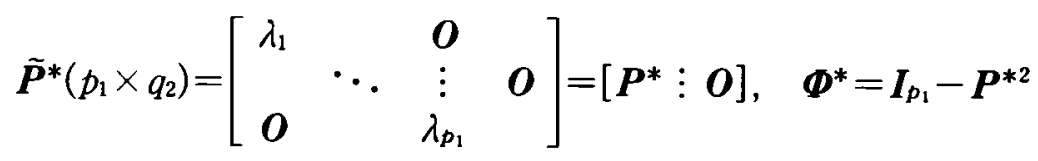

and $\boldsymbol{\Omega}^{*}=\boldsymbol{\Phi}^{*-1} \tilde{\boldsymbol{P}}^{*} \tilde{\boldsymbol{W}}_{44 \cdot 3} \tilde{\boldsymbol{P}}^{*^{\prime}}$.

PROOF. We put

$$
\begin{gathered}
\Sigma_{z z}=\left[\begin{array}{lll}
\Sigma_{11} & \Sigma_{13} & \Sigma_{14} \\
\Sigma_{31} & \Sigma_{33} & \Sigma_{34} \\
\Sigma_{41} & \Sigma_{43} & \Sigma_{44}
\end{array}\right], \quad W_{z z}=\left[\begin{array}{lll}
W_{11} & W_{13} & W_{14} \\
W_{31} & W_{33} & W_{34} \\
W_{41} & W_{43} & W_{44}
\end{array}\right], \\
\Sigma_{2 z}=\left[\Sigma_{21}, \Sigma_{23}, \Sigma_{24}\right], \quad \Sigma_{z 2}=\Sigma_{2 z}^{\prime}, \\
W_{2 z}=\left[W_{21}, W_{23}, W_{24}\right] \text { and } W_{z 2}=W_{2 z}^{\prime}
\end{gathered}
$$

Since $\left[\begin{array}{ll}\boldsymbol{W}_{22} & \boldsymbol{W}_{2 z} \\ \boldsymbol{W}_{z 2} & \boldsymbol{W}_{z z}\end{array}\right]$ is distributed according to $W_{p+q}\left(n,\left[\begin{array}{ll}\boldsymbol{\Sigma}_{22} & \boldsymbol{\Sigma}_{2 z} \\ \boldsymbol{\Sigma}_{z 2} & \boldsymbol{\Sigma}_{z z}\end{array}\right]\right)$, when $\boldsymbol{W}_{z z}$ is given, $\boldsymbol{W}_{22 \cdot 134}$ and $\boldsymbol{W}_{z z}^{-1} \boldsymbol{W}_{z 2}$ are conditionally independently distributed according to $W_{p_{2}}\left(n-p_{1}-q, \Sigma_{22 \cdot 1}\right)$ and $N\left(\Sigma_{z z}^{-1} \Sigma_{z z}, W_{z z}^{-1} \otimes \Sigma_{22 \cdot 1}\right)$, respectively.

Noting that 


$$
W_{z z}^{-1} W_{z 2}=\left[\begin{array}{l}
W_{11 \cdot v}^{-1} W_{12 \cdot v} \\
W_{v v \cdot 1}^{-1} W_{v 2 \cdot 1}
\end{array}\right] \text { and } \Sigma_{z z}^{-1} \Sigma_{z 2}=\left[\begin{array}{c}
\Sigma_{11 \cdot v}^{-1} \Sigma_{12 \cdot v} \\
0
\end{array}\right]
$$

we can see that $\boldsymbol{W}_{v v \cdot 1}^{-1 / 2} \boldsymbol{W}_{v 2 \cdot 1}$ is distributed according to $N\left(\boldsymbol{O}, \boldsymbol{I}_{q} \otimes \boldsymbol{\Sigma}_{22 \cdot 1}\right)$, independently of $\boldsymbol{W}_{22 \cdot 134}$ when $\boldsymbol{W}_{2 z}$ is given.

Since $\Lambda_{11}$ can be written as

$$
\Lambda_{11}=\left|\boldsymbol{W}_{22 \cdot 134}\right| /\left|\boldsymbol{W}_{22 \cdot 134}+\boldsymbol{W}_{2 v \cdot 1} \boldsymbol{W}_{v v \cdot 1}^{-1} \boldsymbol{W}_{v 2 \cdot 1}\right|,
$$

$\Lambda_{11}$ is conditionally distributed according to $\Lambda\left(p_{2}, q, n-p_{1}-q\right)$ when $W_{z z}$ is given. Because the conditional distribution of $\Lambda_{11}$ does not depend on fixed $W_{z z}, \Lambda_{11}$ and $W_{z z}$ are independently distributed. Because $\Lambda_{12}$ is a function of $W_{z z}$, it is seen that $\Lambda_{11}$ and $\Lambda_{12}$ are independently distributed.

Next we prove (iii). First we consider the case of $q_{2} \leq p_{1}$. Then, there exists a $q_{2} \times q_{2}$ orthogonal matrix $\boldsymbol{H}$ and a $p_{1} \times p_{1}$ orthogonal matrix $\boldsymbol{Q}$ such that

$$
H \Sigma_{44 \cdot 3}^{-1 / 2} \Sigma_{41 \cdot 3} \Sigma_{11 \cdot 3}^{-1 / 2} Q^{\prime}=\tilde{P} \text {. }
$$

If we put $\boldsymbol{D}_{\mathbf{4}}=\boldsymbol{H} \boldsymbol{\Sigma}_{44.3}^{-1 / 2}$ and $\boldsymbol{D}_{1}=\boldsymbol{Q} \boldsymbol{\Sigma}_{11.3}^{-1 / 2}$, then it follows that $\boldsymbol{D}_{\mathbf{4}} \boldsymbol{\Sigma}_{44.3} \boldsymbol{D}_{4}^{\prime}=\boldsymbol{I}_{q_{2}}$, $D_{1} \Sigma_{11 \cdot 3} D_{1}^{\prime}=I_{p_{1}}$ and $D_{4} \Sigma_{41 \cdot 3} D_{1}^{\prime}=\tilde{P}$. Hence

$$
\left[\begin{array}{ll}
\tilde{W}_{11 \cdot 3} & \tilde{W}_{14 \cdot 3} \\
\tilde{W}_{41 \cdot 3} & \tilde{W}_{44 \cdot 3}
\end{array}\right]=\left[\begin{array}{cc}
D_{1} & O \\
O & D_{4}
\end{array}\right]\left[\begin{array}{ll}
W_{11 \cdot 3} & W_{14 \cdot 3} \\
W_{41 \cdot 3} & W_{44 \cdot 3}
\end{array}\right]\left[\begin{array}{cc}
D_{1}^{\prime} & O \\
O & D_{4}^{\prime}
\end{array}\right]
$$

is distributed according to

$$
W_{p_{1}+q_{2}}\left(n-q_{1},\left[\begin{array}{cc}
\boldsymbol{I}_{p_{1}} & \widetilde{\boldsymbol{P}}^{\prime} \\
\tilde{\boldsymbol{P}} & \boldsymbol{I}_{q_{2}}
\end{array}\right]\right)
$$

From this, $\tilde{\boldsymbol{W}}_{11 \cdot 3}$ is distributed according to $W_{p_{1}}\left(n-q_{1}, \boldsymbol{I}_{p_{1}}\right)$, and $\tilde{\boldsymbol{W}}_{44 \cdot 3}$ and $\tilde{W}_{44.3} \tilde{W}_{11.3}^{-1} \tilde{W}_{14 \cdot 3}$ are independently distributed according to $W_{q_{2}}\left(n-p_{1}-q_{1}, \boldsymbol{\Phi}\right)$ and $W_{q_{2}}\left(p_{1}, \boldsymbol{\Phi}, \boldsymbol{\Omega}\right)$, respectively when $\tilde{\boldsymbol{W}}_{11 \cdot 3}$ is given. Since $\Lambda_{12}$ can be expressed as

$$
\Lambda_{12}=\left|\tilde{W}_{44 \cdot 13}\right| /\left|\tilde{W}_{44 \cdot 13}+\tilde{W}_{41 \cdot 3} \tilde{W}_{11 \cdot 3}^{-1} \tilde{W}_{14 \cdot 3}\right|,
$$

$\Lambda_{12}$ is conditionally distributed according to $\Lambda\left(q_{2}, p_{1}, n-p_{1}-q_{1}, \boldsymbol{\Omega}\right)$. When $q_{2}>$ $p_{1}$, by expressing $\Lambda_{12}$ as

$$
\Lambda_{12}=\left|\tilde{W}_{11 \cdot 34}\right| /\left|\tilde{W}_{11 \cdot 34}+\tilde{W}_{14 \cdot 3} \tilde{W}_{44 \cdot 3}^{-1} \tilde{W}_{41 \cdot 3}\right|
$$

we can obtain the result in similar way as the case of $q_{2} \leq p_{1}$.

It is necessary to make sure that Lemma 1 coincides with Theorem 1 (ii) when $\Sigma_{41 \cdot 3}=\boldsymbol{O}$ and $\Sigma_{2 v \cdot 1}=\boldsymbol{O}$. When $q_{2} \leq p_{1}$, Lemma 1 clearly coincides with Theorem 1 (ii). When $q_{2}>p_{1}$, putting $\boldsymbol{\Sigma}_{41 \cdot 3}=\boldsymbol{O}$ in Lemma $1, \Lambda_{12}$ is distributed according to $\Lambda\left(q_{2}, p_{1}, n-q\right) . \Lambda\left(q_{2}, p_{1}, n-q\right)$ and $\Lambda\left(p_{1}, q_{2}, n-q\right)$ however, are the same distributions.

We can write the likelihood ratio criterion as

$$
\lambda^{\frac{2}{N}}=\Lambda_{1}=\frac{\left|\boldsymbol{W}_{44 \cdot 123}\right|}{\left|\boldsymbol{W}_{44 \cdot 3}\right|} \times \frac{\left|\boldsymbol{W}_{22 \cdot 13}\right|}{\left|\boldsymbol{W}_{22 \cdot 1}\right|}=\Lambda_{11}^{*} \times \Lambda_{12}^{*} .
$$


Then, concerning the distributions of $\Lambda_{11}^{*}$ and $\Lambda_{12}^{*}$ under $\Sigma_{4 u \cdot 3}=\boldsymbol{O}$, we can obtain a result similar to Lemma 1.

Using Lemma 1 , we obtain the $h$-th moment of $\Lambda_{1}$ defined by (2.3) when $\Sigma_{2 v \cdot 1}=\boldsymbol{O}$.

THEOREM 2. When $\boldsymbol{\Sigma}_{2 v \cdot 1}=\boldsymbol{O}$, the h-th moment of $\Lambda_{1}$ is $E\left(\Lambda_{1}^{h}\right)$

$$
\begin{aligned}
= & \frac{\Gamma_{p_{2}}\left[\frac{1}{2}\left(n-p_{1}-q\right)+h\right] \Gamma_{p_{2}}\left[\frac{1}{2}\left(n-p_{1}\right)\right] \Gamma_{q_{2}}\left[\frac{1}{2}\left(n-p_{1}-q_{1}\right)+h\right] \Gamma_{q_{2}}\left[\frac{1}{2}\left(n-q_{1}\right)\right]}{\Gamma_{p_{2}}\left[\frac{1}{2}\left(n-p_{1}-q\right)\right] \Gamma_{p_{2}}\left[\frac{1}{2}\left(n-p_{1}\right)+h\right] \Gamma_{q_{2}}\left[\frac{1}{2}\left(n-p_{1}-q_{1}\right)\right] \Gamma_{q_{2}}\left[\frac{1}{2}\left(n-q_{1}\right)+h\right]} \\
& \cdot\left|I_{s}-\boldsymbol{L}^{2}\right|^{1 / 2\left(n-q_{1}\right)_{2} F_{1}}\left(\frac{1}{2}\left(n-q_{1}\right), \frac{1}{2}\left(n-q_{1}\right) ; \frac{1}{2}\left(n-q_{1}\right)+h ; \boldsymbol{L}^{2}\right),
\end{aligned}
$$

where $s=\min \left(q_{2}, p_{1}\right), \lambda_{1}^{2}, \cdots, \lambda_{s}^{2}, \cdots$ are the ordered latent roots of $\Sigma_{44 \cdot 3}^{-1} \Sigma_{41 \cdot 3} \Sigma_{11 \cdot 3}^{-1}$ $\boldsymbol{\Sigma}_{14 \cdot 3,} \boldsymbol{L}^{2}=\operatorname{diag}\left(\lambda_{1}^{2}, \cdots, \lambda_{s}^{2}\right), \Gamma_{p_{2}}[\cdot]$ and $\Gamma_{q_{2}}[\cdot]$ are multivariate gamma functions and ${ }_{2} F_{1}$ is a hypergeometric function of matrix arguments.

Proof. From Lemma 1 (i),

$$
E\left(\Lambda_{1}^{h}\right)=E\left(\Lambda_{11}^{h}\right) E\left(\Lambda_{12}^{h}\right)
$$

Using Lemma 1 (ii), (iii) and Theorem 11.2.6 of Muirhead [6], we get

$$
\begin{aligned}
E\left(\Lambda_{11}^{h}\right)= & \frac{\Gamma_{p_{2}}\left[\frac{1}{2}\left(n-p_{1}-q\right)+h\right] \Gamma_{p_{2}}\left[\frac{1}{2}\left(n-p_{1}\right)\right]}{\Gamma_{p_{2}}\left[\frac{1}{2}\left(n-p_{1}-q\right)\right] \Gamma_{p_{2}}\left[\frac{1}{2}\left(n-p_{1}\right)+h\right]}, \\
E\left(\Lambda_{12}^{h}\right)= & \frac{\Gamma_{q_{2}}\left[\frac{1}{2}\left(n-p_{1}-q_{1}\right)+h\right] \Gamma_{q_{2}}\left[\frac{1}{2}\left(n-q_{1}\right)\right]}{\Gamma_{q_{2}}\left[\frac{1}{2}\left(n-p_{1}-q_{1}\right)\right] \Gamma_{q_{2}}\left[\frac{1}{2}\left(n-q_{1}\right)+h\right]} \\
& \cdot\left|\boldsymbol{I}_{q_{2}}-\boldsymbol{P}^{2}\right|^{1 / 2\left(n-q_{1}\right)}{ }_{2} F_{1}\left(\frac{1}{2}\left(n-q_{1}\right), \frac{1}{2}\left(n-q_{1}\right) ; \frac{1}{2}\left(n-q_{1}\right)+h ; \boldsymbol{P}^{2}\right), q_{2} \leq p_{1}, \\
E\left(\Lambda_{12}^{h}\right)= & \frac{\Gamma_{p_{1}}\left[\frac{1}{2}(n-q)+h\right] \Gamma_{p_{1}}\left[\frac{1}{2}\left(n-q_{1}\right)\right]}{\Gamma_{p_{1}}\left[\frac{1}{2}(n-q)\right] \Gamma_{p_{1}}\left[\frac{1}{2}\left(n-q_{1}\right)+h\right]} \\
& \cdot\left|\boldsymbol{I}_{p_{1}}-\boldsymbol{P}^{* 2}\right|^{1 / 2\left(n-q_{1}\right)}{ }_{2} F_{1}\left(\frac{1}{2}\left(n-q_{1}\right), \frac{1}{2}\left(n-q_{1}\right) ; \frac{1}{2}\left(n-q_{1}\right)+h ; \boldsymbol{P}^{* 2}\right), q_{2}>p_{1},
\end{aligned}
$$

where $\boldsymbol{P}$ and $\boldsymbol{P}^{*}$ are defined in Theorem 3.

Since

$$
\frac{\Gamma_{q_{2}}\left[\frac{1}{2}\left(n-p_{1}-q_{1}\right)+h\right] \Gamma_{q_{2}}\left[\frac{1}{2}\left(n-q_{1}\right)\right]}{\Gamma_{q_{2}}\left[\frac{1}{2}\left(n-p_{1}-q_{1}\right)\right] \Gamma_{q_{2}}\left[\frac{1}{2}\left(n-q_{1}\right)+h\right]}=\frac{\Gamma_{p_{1}}\left[\frac{1}{2}(n-q)+h\right] \Gamma_{p_{1}}\left[\frac{1}{2}\left(n-q_{1}\right)\right]}{\Gamma_{p_{1}}\left[\frac{1}{2}(n-q)\right] \Gamma_{p_{1}}\left[\frac{1}{2}\left(n-q_{1}\right)+h\right]},
$$


we can write as

$$
\begin{aligned}
E\left(\Lambda_{12}^{h}\right)= & \frac{\Gamma_{q_{2}}\left[\frac{1}{2}\left(n-p_{1}-q_{1}\right)+h\right] \Gamma_{q_{2}}\left[\frac{1}{2}\left(n-q_{1}\right)\right]}{\Gamma_{q_{2}}\left[\frac{1}{2}\left(n-p_{1}-q_{1}\right)\right] \Gamma_{q_{2}}\left[\frac{1}{2}\left(n-q_{1}\right)+h\right]} \\
& \cdot \mid I_{s}-L^{2 \mid 1 / 2\left(n-q_{1}\right)}{ }_{2} F_{1}\left(\frac{1}{2}\left(n-q_{1}\right), \frac{1}{2}\left(n-q_{1}\right) ; \frac{1}{2}\left(n-q_{1}\right)+h ; L^{2}\right) .
\end{aligned}
$$

By expressing $\Lambda_{1}$ as (3.2), we similarly have the following theorem.

THEOREM 3. When $\Sigma_{4 u \cdot 3}=\boldsymbol{O}$, the h-th moment of $\Lambda_{1}$ is

$$
\begin{aligned}
& E\left(\Lambda_{1}^{h}\right) \\
& =\frac{\Gamma_{q_{2}}\left[\frac{1}{2}\left(n-p-q_{1}\right)+h\right] \Gamma_{q_{2}}\left[\frac{1}{2}\left(n-q_{1}\right)\right] \Gamma_{p_{2}}\left[\frac{1}{2}\left(n-p_{1}-q_{1}\right)+h\right] \Gamma_{p_{2}}\left[\frac{1}{2}\left(n-p_{1}\right)\right]}{\Gamma_{q_{2}}\left[\frac{1}{2}\left(n-p-q_{1}\right)\right] \Gamma_{q_{2}}\left[\frac{1}{2}\left(n-q_{1}\right)+h\right] \Gamma_{p_{2}}\left[\frac{1}{2}\left(n-p_{1}-q_{1}\right)\right] \Gamma_{p_{2}}\left[\frac{1}{2}\left(n-p_{1}\right)+h\right]} \\
& \cdot\left|\boldsymbol{I}_{s}-\tilde{\boldsymbol{L}}^{2}\right|^{1 / 2\left(n-p_{1}\right)}{ }_{2} F_{1}\left(\frac{1}{2}\left(n-p_{1}\right), \frac{1}{2}\left(n-p_{1}\right) ; \frac{1}{2}\left(n-p_{1}\right)+h ; \tilde{\boldsymbol{L}}^{2}\right),
\end{aligned}
$$

where $r=\min \left(p_{2}, q_{1}\right), \tilde{\lambda}_{1}^{2}, \cdots, \tilde{\lambda}_{r}^{2}, \cdots$ are the ordered latent roots of $\Sigma_{22 \cdot 1}^{-1} \Sigma_{23 \cdot 1} \Sigma_{33 \cdot 1}^{-1}$ $\Sigma_{32 \cdot 1}$ and $\tilde{\boldsymbol{L}}^{2}=\operatorname{diag}\left(\tilde{\lambda}_{1}^{2}, \cdots, \bar{\lambda}_{r}^{2}\right)$.

\section{Asymptotic expansions of the non-null distributions}

From Theorem 1 (iii), when $H$ is true, the characteristic function of $-2 \rho \log \lambda$ is given by

$$
\phi(t)=(1-2 i t)^{-\frac{f}{2}}+O\left(M^{-2}\right)
$$

where $f=p q-p_{1} q_{1}$

$$
\rho=1-\frac{1}{2 N}\left\{p+q+3-\frac{1}{f} p_{1} q_{1}\left(p_{2}+q_{2}\right)\right\}
$$

and $M=\rho N$.

We consider the distribution of $-2 \rho \log \lambda$ under the sequence of local alternatives

$$
K_{M}: \Sigma_{2 v \cdot 1}=O, \quad L^{2}=\frac{1}{M} \Theta
$$

where $\boldsymbol{L}^{2}$ is defined in Theorem 2 and $\boldsymbol{\Theta}=\operatorname{diag}\left(\theta_{1}, \cdots, \theta_{s}\right)$ is fixed. Let $\phi(t, \boldsymbol{\theta})$ be the characteristic function of the distribution of $-2 \rho \log \lambda$ under $K_{M}$. From Theorem $2, \phi(t, \boldsymbol{\theta})$ can be computed as 
(4.3) $\phi(t, \boldsymbol{\Theta})$

$$
\begin{aligned}
& =E\left(\lambda^{-2 i t p}\right) \\
& =E\left(\Lambda_{1}^{- \text {Mit }}\right) \\
& =\frac{\left(\begin{array}{c}
\Gamma_{p_{2}}\left[\frac{1}{2}\left(n-p_{1}-q\right)-M i t\right] \Gamma_{p_{2}}\left[\frac{1}{2}\left(n-p_{1}\right)\right] \\
\cdot \Gamma_{q_{2}}\left[\frac{1}{2}\left(n-p_{1}-q_{1}\right)-M i t\right] \Gamma_{q_{2}}\left[\frac{1}{2}\left(n-q_{1}\right)\right]
\end{array}\right)}{\left(\begin{array}{c}
\Gamma_{p_{2}}\left[\frac{1}{2}\left(n-p_{1}-q\right)\right] \Gamma_{p_{2}}\left[\frac{1}{2}\left(n-p_{1}\right)-M i t\right] \\
\cdot \Gamma_{q_{2}}\left[\frac{1}{2}\left(n-p_{1}-q_{1}\right)\right] \Gamma_{q_{2}}\left[\frac{1}{2}\left(n-q_{1}\right)-M i t\right]
\end{array}\right)} \\
& \cdot\left|\boldsymbol{I}_{s}-\frac{1}{M} \Theta\right|_{2}^{1 / 2\left(n-q_{1}\right)}{ }_{2} F_{1}\left(\frac{1}{2}\left(n-q_{1}\right), \frac{1}{2}\left(n-q_{1}\right) ; \frac{1}{2}\left(n-q_{1}\right)-M i t ; \frac{1}{M} \Theta\right) \\
& =\phi(t)\left|\boldsymbol{I}_{s}-\frac{1}{M} \Theta\right|^{1 / 2\left(n-q_{1}\right)} \\
& \cdot{ }_{2} F_{1}\left(\frac{1}{2}\left(n-q_{1}\right), \frac{1}{2}\left(n-q_{1}\right) ; \frac{1}{2}\left(n-q_{1}\right)-M i t ; \frac{1}{M} \Theta\right) \\
& =\phi(t)\left|\boldsymbol{I}_{s}-\frac{1}{M} \Theta\right|^{1 / 2(M+\delta)} \\
& \cdot{ }_{2} F_{1}\left(\frac{1}{2}(M+\delta), \frac{1}{2}(M+\delta) ; \frac{1}{2} M(1-2 i t)+\frac{1}{2} \delta ; \frac{1}{M} \Theta\right),
\end{aligned}
$$

where

$$
\delta=\frac{1}{2}\left\{p+q_{2}-q_{1}+1-\frac{1}{f} p_{1} q_{1}\left(p_{2}+q_{2}\right)\right\}
$$

Using Theorem 8.2.14 from Muirhead [6], we have

$$
\begin{aligned}
\mid \boldsymbol{I}_{s} & -\left.\frac{1}{M} \Theta\right|_{2} ^{1 / 2(M+\delta)} F_{1}\left(\frac{1}{2}(M+\delta), \frac{1}{2}(M+\delta) ; \frac{1}{2} M(1-2 i t)+\frac{1}{2} \delta ; \frac{1}{M} \boldsymbol{\theta}\right) \\
= & \exp \left(\frac{i t \sigma_{1}}{1-2 i t}\right)\left[1-\frac{1}{4 M}\left\{\sigma_{2}+2 \delta \sigma_{1}-\frac{4 \delta \sigma_{1}}{1-2 i t}-\frac{2\left(\sigma_{2}-\delta \sigma_{1}\right)}{(1-2 i t)^{2}}\right.\right. \\
& \left.\left.+\frac{\sigma_{2}}{(1-2 i t)^{3}}\right\}\right]+O\left(M^{-2}\right),
\end{aligned}
$$

where $\sigma_{1}=\operatorname{tr} \Theta$ and $\sigma_{2}=\operatorname{tr} \Theta^{2}$.

From (4.1), (4.3) and (4.5), we can expand $\phi(t, \Theta)$ as

$$
\begin{aligned}
\phi(t, \boldsymbol{\Theta})= & (1-2 i t)^{-\frac{f}{2}} \exp \left(\frac{i t \sigma_{1}}{1-2 i t}\right)\left[1-\frac{1}{4 M}\left\{\sigma_{2}+2 \delta \sigma_{1}-\frac{4 \delta \sigma_{1}}{1-2 i t}\right.\right. \\
& \left.\left.-\frac{2\left(\sigma_{2}-\delta \sigma_{1}\right)}{(1-2 i t)^{2}}+\frac{\sigma_{2}}{(1-2 i t)^{3}}\right\}\right]+O\left(M^{-2}\right)
\end{aligned}
$$

Inverting (4.6), we obtain the following theorem.

THEOREM 4. Under $K_{M}$ defined by (4.2), the distribution of $-2 \rho \log \lambda$ can be asymptotically approximated by 


$$
\begin{aligned}
\operatorname{Pr}( & -2 \rho \log \lambda \leq x) \\
= & \operatorname{Pr}\left(\chi_{f}^{2}\left(\sigma_{1}\right) \leq x\right)-\frac{1}{4 M}\left\{\left(\sigma_{2}+2 \delta \sigma_{1}\right) \operatorname{Pr}\left(\chi_{f}^{2}\left(\sigma_{1}\right) \leq x\right)\right. \\
& -4 \delta \sigma_{1} \operatorname{Pr}\left(\chi_{f+2}^{2}\left(\sigma_{1}\right) \leq x\right)-2\left(\sigma_{2}-\delta \sigma_{1}\right) \operatorname{Pr}\left(\chi_{f+4}^{2}\left(\sigma_{1}\right) \leq x\right) \\
& \left.+\sigma_{2} \operatorname{Pr}\left(\chi_{f+6}^{2}\left(\sigma_{1}\right) \leq x\right)\right\}+O\left(M^{-2}\right),
\end{aligned}
$$

where $\sigma_{1}=\operatorname{tr} \Theta, \sigma_{2}=\operatorname{tr} \Theta^{2}, \chi_{f}^{2}(\sigma)$ is a noncetral $\chi^{2}$-variate with degrees of freedom $f$ and noncentrality parameter $\sigma$, and $\delta$ is defined by (4.4).

Similarly, we can obtain the asymptotic expansion of the distribution of $-2 \rho \log \lambda$ under the sequence of local alternatives

$$
\tilde{K}_{M}: \Sigma_{4 u \cdot 3}=\boldsymbol{O}, \quad \tilde{L}^{2}=\frac{1}{M} \tilde{\Theta}
$$

where $\tilde{\boldsymbol{L}}$ is defined in Lemma 3 and $\widetilde{\boldsymbol{\Theta}}=\operatorname{diag}\left(\tilde{\theta}_{1}, \cdots, \tilde{\theta}_{r}\right)$ is fixed. The result is following.

THEOREM 5. Under $\tilde{K}_{M}$ defined by (4.8), the distribution of $-2 \rho \log \lambda$ can be asymptotically approximated by

$$
\begin{aligned}
\operatorname{Pr}( & -2 \rho \log \lambda \leq x) \\
= & \operatorname{Pr}\left(\chi_{f}^{2}\left(\tilde{\sigma}_{1}\right) \leq x\right)-\frac{1}{4 M}\left\{\left(\tilde{\sigma}_{2}+2 \tilde{\delta} \tilde{\sigma}_{1}\right) \operatorname{Pr}\left(\chi_{f}^{2}\left(\tilde{\sigma}_{1}\right) \leq x\right)\right. \\
& -4 \tilde{\delta}_{1} \operatorname{Pr}\left(\chi_{f+2}^{2}\left(\tilde{\sigma}_{1}\right) \leq x\right)-2\left(\tilde{\sigma}_{2}-\tilde{\delta} \tilde{\sigma}_{1}\right) \operatorname{Pr}\left(\chi_{f+4}^{2}\left(\tilde{\sigma}_{1}\right) \leq x\right) \\
& \left.+\tilde{\sigma}_{2} \operatorname{Pr}\left(\chi_{f+6}^{2}\left(\tilde{\sigma}_{1}\right) \leq x\right)\right\}+O\left(M^{-2}\right),
\end{aligned}
$$

where $\tilde{\sigma}_{1}=\operatorname{tr} \tilde{\boldsymbol{\Theta}}, \tilde{\sigma}_{2}=\operatorname{tr} \tilde{\Theta}^{2}$ and

$$
\tilde{\delta}=\frac{1}{2}\left\{p_{2}-p_{1}+q+1-\frac{1}{f} p_{1} q_{1}\left(p_{2}+q_{2}\right)\right\} .
$$

The following theorem follows from Lemma 3.1 of Fujikoshi [3] using Theorems 4 and 5 described in this paper.

THEOREM 6. Let $\beta(\boldsymbol{\Theta})$ and $\beta(\tilde{\boldsymbol{\Theta}})$ be the power functions of the test based on $-2 \rho \log \lambda$ with a level of significance $\alpha$ under $K_{M}$ and $\tilde{K}_{M}$, respectively. Then $\beta(\boldsymbol{\Theta})$ and $\beta(\tilde{\boldsymbol{\Theta}})$ can be expanded as

$$
\begin{aligned}
& \beta(\boldsymbol{\Theta})=\operatorname{Pr}\left(\chi_{f}^{2}\left(\sigma_{1}\right) \geq u\right)+\frac{1}{2 M} \sum_{i=1}^{3} c_{i} g_{f+2 i}\left(u ; \sigma_{1}\right)+o\left(M^{-1}\right), \\
& \beta(\tilde{\boldsymbol{\Theta}})=\operatorname{Pr}\left(\chi_{f}^{2}\left(\tilde{\sigma}_{1}\right) \geq u\right)+\frac{1}{2 M} \sum_{i=1}^{3} \tilde{c}_{i} g_{f+2 i}\left(u ; \tilde{\sigma}_{1}\right)+o\left(M^{-1}\right),
\end{aligned}
$$

where $u$ is the $\alpha$ point of $\chi_{f}^{2}, g_{f}(u ; \sigma)$ is the p.d.f. of a noncentral $\chi^{2}$-variate $\chi_{f}^{2}(\sigma)$ with $f$ degrees of freedom and noncentrality parameter $\sigma, c_{1}=\sigma_{2}+2 \delta \sigma_{1}, c_{2}=\sigma_{2}$ $-2 \delta \sigma_{1}, c_{3}=-\sigma_{2}, \tilde{c}_{1}=\tilde{\sigma}_{2}+2 \delta \tilde{\sigma}_{1}, \tilde{c}_{2}=\tilde{\sigma}_{2}-2 \tilde{\delta} \tilde{\sigma}_{1}$ and $\tilde{c}_{3}=-\tilde{\sigma}_{2}$.

Note that the power function $\beta(\Theta)$ is a power function that is not under the general alternative but under the local alternatives $K_{M}$ which correspond to the intermediate hypothesis $H_{1}$ defined by (3.1). When $x_{2}$ can be regarded as a 
redundant variable(s) in CCA between $x_{u}$ and $x_{v}$ based on the likelihood ratio statistic $\Lambda_{12}$ for $H_{1}$ v.s. $K$, we are interested in the simultaneous redundancy of $x_{2}$ and $x_{4}$ in CCA between $x_{u}$ and $x_{v}$. To solve this, we perform a test of $H$ v.s. $K$ where the power of the likelihood ratio test can be approximated by $\beta(\Theta)$.

\section{Acknowledgements}

The authors would like to thank the referees for their valuable comments.

\section{REFERENCES}

[1] Anderson, T. W. (1984). An Introduction to Multivariate Statistical Analysis. 2nd ed., Wiley, New York.

[2] Fujikoshi, Y. (1982). A test for additional information in canonical correlation analysis, Ann. Inst. Statist. Math., 34, 523-530.

[3] Fujikoshi, Y. (1988). Comparison of powers of a class of tests for multivariate linear hypotheses and independence, J. Multiv. Anal., 26, 48-58.

[4] Kariya, T., Fujikoshi, Y. and Krishnaiah, P. R. (1987). On tests for selection of variables and independence under multivariate regression models, J. Multi. Anal., 21, 207-237.

[5] McKay, R. J. (1977). Variable selection in multivariate regression: An application of simultaneous procedure, J. Roy. Statist. Soc. B, 39, 371-380.

[6] Murhead, R. J. (1982). Aspects of Multivariate Statistical Theory, Wiley, New York. 